APC Alignment - 9 species with edits (2018)

\author{
Dimerization \\ PHA03307 \\ DLG (Axin) Binding Site \\ Basic Region \\ CLUSTAL O(1.2.4) multiple sequence alignment \\ XP_783363.3 - Strongylocentrotus purpuratus \\ NP_001137312.1 - Danio rerio \\ NP_001084351.1 - Xenopus laevis \\ XP_004949340.1 - Gallus gallus \\ XP_007497871.1 - Monodelphis domestica \\ NP_031488.2 - Mus musculus \\ NP_001069454.2 - Bos taurus \\ XP_014996065.1 - Macaca mulatta \\ AAA03586.1 - Homo sapiens
}

Beta Catenin and SAMP Repeats (one)(two three)

MAP65_ASE1 Microtubule Association Family (basic region for microtubule binding)

$$
\begin{aligned}
& \text { XP_783363.3 } \\
& \text { NP_0 } 01137312.1 \\
& \text { NP_0 01084351.1 } \\
& \text { XP_0 04949340.1 } \\
& \text { XP_0 0 } 497871.1 \\
& \text { NP_031488.2 } \\
& \text { NP_001069454.2 } \\
& \text { XP_014996065.1 } \\
& \text { AAAD03586.1 }
\end{aligned}
$$

$\mathrm{XP}+783363.3$

NP 001137312.1

NP_001084351.1

$\mathrm{XP}-004949340.1$

XP_007497871.1

NP 031488.2

$\mathrm{NP}-001069454.2$

$\mathrm{XP}-014996065.1$

AAĀ03586.1

XP_783363.3

NP_001137312.1

NP 001084351.1

XP_004949340.1

XP 007497871.1

NP_031488.2

NP_001069454.2

XP_014996065.1

$\mathrm{AA} \overline{\mathrm{A}} 03586.1$

XP 783363.3

NP_001137312.1

NP 001084351.1

XP 004949340.1

XP_007497871.1

NP 031488.2

NP_001069454.2

XP_014996065.1

AA $\bar{A} 03586.1$

XP_783363.3

NP 001137312.1

NP_001084351.1

XP 004949340.1

XP_007497871.1
--MSSYDQLLHQVESLKAENSHLKMELKDNSSOLNKLDSVVGHLQSGKP---GMGEDDLR MAAASYDQLLKQVEALKMENSNLRQELEDNSNHLNKLETEASNMKEVVLKQLQGSIDEDSK MAAASYDQLVKOVEALTMENTNLRQELEDNSNHLTKLETEATNMKEVLKQLQGSIEDEAM MAAASYDQLLKQVEALKMENSNLRQELEDNSNHLTKLETEASNMKEVLKQLQGSIEDEAI MAAASYDQLLKQVEALKMENSNLRQELEDNSNHLTKLETEASNMKEVLKQLQGSIEDEAM MAAASYDQLLKQVEALKMENSNLRQELEDNSNHLTKLETEASNMKEVLKQLQGSIEDETM MAAASYDQLLKQVEALKMENSNLRQELEDNSNHLTKLETEASNMKEVLKQLQGS IEDEAM MAAASYDQLLKQVEALKMENSNLRQELEDNSNHLTKLETEASNMKEVLKQLQGSIEDEAM MAAASYDQLLKQVEALKMENSNLRQELEDNSNHLTKLETEASNMKEVLKQLQGSIEDEAM $: * * * * *:: * * *: * . * *:: *: * *: * * * .: * . * *:: .::::$ *

AQITIHGLAMTTAAASATSSSAATSG---AGPASSFOVNGGAMLPGKPHSLPTS DSOGOIEFLERIKEMSLDPSGF SGVKLRSKASLQGSA---------DSSPSPSP ASSGPIDLLERF KDLNLDSSNIPAGKARPKMSMRSYGSREGSLSGHSGECSPVP ASSGQIDLLERLKELNLESTSFPGVKLROKVSVRSYGSREGSVSSRSGECSPVP ASSGQIDLLERLKELNLDSSNFPGVKLRPKMSIRSYGSREGSVSSRSGECSPVP T-SGQIDLLERLKEFNLD-SNFPGVKLRSKMSLRSYGSREGSVSSRSGECSPVP ASSGOIDLLERLKELNLDSSNFPGVKLRSKMSLRSYGSREGSVSSRSGECSPVP ASSGQIDLLERLKELNLDSSNFPGVKLRSKMSLRSYGSREGSVSSRSGECSPVP ASSGQIDLLERLKELNLDSSNFPGVKLRSKMSLRSYGSREGSVSSRSGECSPVP

$$
\text { * : : : :. . * : . }
$$

LGTSGTDGSLRQTKDHF IRHQELERDE VSSCPRRGASSGGRDSAGYLEELEKE VGSFORRGLLNGSRESAGYMEELEKE MGSFPRRGFMNGSRESTGYLEELEKE MGSFSRRGFMNGSRESTGYLEELEKE MGSFPRRTFVNGSRESTGYLEELEKE MGSFPRRGFVNGSRENTGYLEELEKE MGSFPRRGFVNGSRESTGYLEELEKE MGSFPRRGFVNGSRESTGYLEELEKERSLLLADLDKEEKEKDWYYAQLQNLTKRIDSLPL LILNEQAKEENLRSQCLAQIQSLTRRIEDLPI LLVAELEKEEKEKDWYYAQLQNLTKRIDSLPL LLIAEHEKEEKEKRWYYAOLONLTKRIDSLPL LLLAELEKEEKEKDWYYAQLQNLTKRIDSLPL LLLAELEKEEKEKDWYYAQLQNLTKRIDSLPL LLLADLDKEEKEKDWYYAQLQNLTKRIDSLPL LLLADLDKEEKEKDWYYAOLONLTKRIDSLPL LLLADLDKEEKEKDWYYAQLQNLTKRIDSLPL

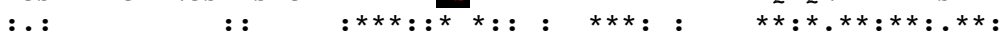

TDNYSLOTDMSRROLEYEAROLRELMOERLGSMEDIAARHRMRMORLSAIEYELROVOEO

QQQHQQQQQQQQQQQQQ $Q Q Q Q Q Q Q Q M L A K V W E Q G A$ 
NP_031488.2

NP_001069454.2

XP_014996065.1 AA $\bar{A} 03586.1$

XP_783363.3

NP_001137312.1

NP 001084351.1

XP_004949340.1

XP 007497871.1

NP_031488.2

NP_001069454.2

XP 014996065.1

AAA 03586.1

XP 783363.3

NP_001137312.1

NP_001084351.1

XP_004949340.1

XP_007497871.1

NP 031488.2

NP_001069454.2

XP_014996065.1

AAĀ03586.1

XP_783363.3

NP 001137312.1

NP_001084351.1

XP_004949340.1

XP 007497871.1

NP_031488.2

NP 001069454.2

XP_014996065.1

AA $\bar{A} 03586.1$

XP 783363.3

NP_001137312.1

NP 001084351.1

XP_004949340.1

XP_007497871.1

NP_031488.2

NP_001069454.2

$\mathrm{XP}-014996065.1$

AAĀ03586. 1

XP_783363.3

NP 001137312.1

NP_001084351.1

XP 004949340.1

XP 007497871.1

NP_031488.2

NP 001069454.2

XP_014996065.1

AA $\bar{A} 03586.1$

XP 783363.3

NP_001137312.1

NP 001084351.1

XP_004949340.1

XP 007497871.1

NP 031488.2

NP_001069454.2

XP- 014996065.1

AA $\bar{A} 03586.1$
LOSOAAE-AERSSOSRHDAASHEAGROHEGHGVAE--SNTA-ASSSGOSPATRVDHETAS LQSQATE-AERSSQSKHEAGSHEAERQNEGQGVAE--INMA-TSGSGQGSTTRIDHETAS LQSOATE-AERS SQNKHETGSHDAERQNEGQGVAE--INMA-TSGNGQGSTTRMDHETAS LQSQATE-AERSSQNKHETGSHDAERQNEGQGVGE--INMA-TSGNGQGSTTRMDHETAS * : : .

:

EMSSAGSYSVPRRLTSHLGTK

GFVYSLLSMLGSHDRDDMASTLLMMSRSADSCLAMRO EMVYSLLSMLGTHDKDDMSRTLLAMSSSODSCIAMROS VMSSNSTYSVPRRLTSHLGT VMSSSNNYSVPRRLTSHLGT GGSANGAYSVPRRLTSHLGT VLSSSGTHSAPRRLTSHLG' VLSSSSTHSAPRRLTSHLGT VLSSSSTHSAPRRLTSHLG VLSSSSTHSAPRRLTSHLG? EMVYSLLSMLGTHDKDDMSRTLLAMSSSODSCIAMROS EMVYSLLSMLGTHDKDDMSRTLLAMSSSQDSCIAMRQS EMVYSLLSMLGTHDKDDMSRTLLAMSSSODSCISMROS EMVYSLLSMLGTHDKDDMSRTLLAMSSSODSCISMRQS EMVYSLLSMLGTHDKDDMSRTLLAMSSSODSCISMRQS EMVYSLLSMLGTHDKDDMSRTLLAMSSSQDSCISMRQS $. . *:: *: * * * * * * * * *: * *: * * *: * * * * * * * * *:: * * * *$

CIPLLIHILHGTDQESV-LGNFRGSOKARDCASTALHNIVHLNPDEKRRKOEGRVLRLI GCLPLLIQLLHGNDKDSVLLGNSRGSKEARARASAALHNI I HSQPDDKRGRREIRVLHLL GCLPLLIQLLHGNDKDSVLLGNSRGSKEARASGSAALDNI I HSQPDDKRGRRE IRVLHLL GCLPLLIOLLHGNDKDSVLLGNSRGSKEARARASAALHNI IHSOPDDKRGRRE IRVLHLL GCLPLLIQLLHGNDKDSVLLGNSRGSKEARARASAALHNI IHSQPDDKRGRREIRVLHLL GCLPLLIOLLHGNDKDSVLLGNSRGSKEARARASAALHNI I HSOPDDKRGRRE IRVLHLL GCLPLLIQLLHGNDKDSVLLGNSRGSKEARARASAALHNI I HSQPDDKRGRRE IRVLHLL GCLPLLIQLLHGNDKDSVLLGNSRGSKEARARASAALHNI I HSQPDDKRGRREIRVLHLL

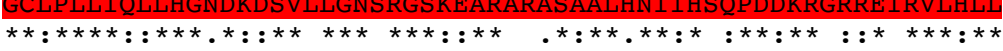

EQIRTYCDSLVEAETKES----- ISSSQNALPIDHNPGPAMAALMKLSFDEEHRSAICHT EQIRAYCETCWEWQESHERGVDQDKN EQIRAYCETCWEWQEAHEQGMDQDKN EQIRAYCETCWEWQEAHEQGMDQDKN EQIRAYCETCWQWQEAHEQGMDQDKN EQIRAYCETCWEWQEAHEQGMDQDKN EQIRAYCETCWEWQEAHEQGMDQDKN EQIRAYCETCWEWQEAHEQGMDQDKN PSPVEHQICPAVCVLMKLSFDEEHRHAMNE APVDHQICPAVCVLMKLSFDEEHRHAMNE PAPVDHQICPAVCVLMKLSFDEEHRHAMNE PAPVEHQICPAVCVLMKLSFDEEHRHAMNE PAPVEHQICPAVCVLMKLSFDEEHRHAMNEI PAPVEHQICPAVCVLMKLSFDEEHRHAMNEI PAPVEHQICPAVCVLMKLSFDEEHRHAMNE

$* * *: * *:: \quad: \quad: \ldots \quad \ldots \quad *:: * \quad * *: \ldots * * * * * * * * * * *: . * *$

412

464

472

470

470

468

470

470

470

GLHAIAELLQVDYEVHGSSNDQYTVTLRRYAGMALTNLTFGDVTNKALLCSMKGCMKALV GLQAIGELLQVDCEIYGLTNDHYSVTLRRYAGMALTNLTFGDVANKATLCSMKGCMRAMV GLQAIAELLQVDCEMYGLINDHYSVTLRRYAGMALTNLTFGDVANKATLCSMKSCMRALV GLOAIAELLOVDCEMYGLTNDHYSVTLRRYAGMALTNLTFGDVANKA TLCSMKGCMRALV GLQAIAELLQVDCEMYGLTSDHYSVTLRRYAGMALTNLTFGDVANKATLCSMKGCMRALV GLQAIAELLQVDCEMYGLTNDHYSVTLRRYAGMALTNLTFGDVANKATLCSMKGCMRALV GLQAIAELLQVDCEMYGLTNDHYS ITLRRYAGMALTNLTFGDVANKATLCSMKGCMRALV GLQAIAELLQVDCEMYGLTNDHYS ITLRRYAGMALTNLTFGDVANKATLCSMKGCMRALV

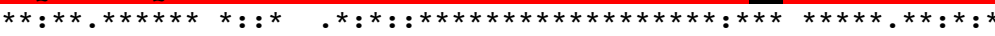

ALLSAESEDLRQVAASVLRNLSWRADMASKKALREAGAVVALMTCSLEVKKESTLKSVLS AQLKSESEDLQDVIASVLRNLSWRADVNSKKILREVGSVRALMECALEVQKESTLKSVLS AQLKSESEDLQOVIASVLRNLSWRADVNSKKTLREVGSVKALMECALDVKKESTLKSVLS AQLKSESEDLQOVIASVLRNLSWRADVNSKKTLREVGSVKALMECALEVKKESTLKSVLS AOLKSESEDLE OVIASVLRNLSWRADVNSKKTLREVGSVKALMECALEVKKE STLKSVLS AQLKSESEDLQ OVIASVLRNLSWRADVNSKKTLREVGSVKALMECALEVKKE STLKSVLS AOLQSESEDLQOVIASVLRNLSWRADVNSKKTLREVGSVKALMECALEVKKESTLKSVLS AQLKSESEDLQDVIASVLRNLSWRADVNSKKTLREVGSVKALMECALEVKKESTLKSVLS AQLKSESEDLQQVIASVLRNLSWRADVNSKKTLREVGSVKALMECALEVKKESTLKSVLS
$* * .: * * * * * * * * * * * * * * * *: * * * * * * *: * * * * *: * *: * * * * * * * *$

ALWNLSAHCTENKADICAVNGALEFLVSSLTYRSPTRNSAVVENGGGILRNVSSHIATSE ALWNLSAHCTENKADICTVPGALAFLVSTLTYRSQTNTLAI IESGGGILRNVSSLIATNE ALWNLSAHCTENKADICSVDGALAFLVSTLTYRSOTNTLAI IESGGGILRNVSSLIATNE ALWNLSAHCTENKADICAVDGALAFLVGTLTYRSQTNTLAI IESGGGILRNVSSLIATNE ALWNLSAHCTENKADICAVDGALAFLVGTLTYRSQTNTLAI IESGGGILRNVSSLIATNE ALWNLSAHCTENKADICAVDGALAFLVGTLTYRSOTNTLAI IESGGGILRNVSSLIATNE ALWNLSAHCTENKADICAVDGALAFLVGTLTYRSQTNTLAI IESGGGILRNVSSLIATNE ALWNLSAHCTENKADICAVDGALAFLVGTLTYRSQTNTLAI IESGGGILRNVSSLIATNE 
XP_783363.3

NP_001137312.1 NP_001084351.1 XP 004949340.1 XP_007497871.1 NP 031488.2

NP_001069454.2 XP 014996065.1 AAĀ03586.1

XP_783363.3 NP_001137312.1 NP_001084351.1 XP_004949340.1 XP_007497871.1 NP_031488.2 NP_001069454.2 XP_014996065.1 $\mathrm{AA} \overline{\mathrm{A}} 03586.1$

XP 783363.3 NP_001137312.1 NP 001084351.1 XP 004949340.1 XP_007497871.1 NP 031488.2 NP_001069454.2 XP_014996065.1 AAĀ03586.1

XP_783363.3 NP 001137312.1 NP_001084351.1 XP_004949340.1 XP 007497871.1 NP_031488.2 NP 001069454.2 XP_014996065.1 AAA 03586.1

XP 783363.3 NP_001137312.1 NP 001084351.1 XP_004949340.1 XP_007497871.1 NP 031488.2 NP_001069454.2 XP 014996065.1 AAA 03586.1

XP_783363.3 NP 001137312.1 NP_001084351.1 XP 004949340.1 $\mathrm{XP}-007497871.1$ NP 031488.2 NP 001069454.2 XP_014996065.1 AA $\bar{A} 03586.1$

XP 783363.3 NP_001137312.1 NP 001084351.1 XP_004949340.1 XP_007497871.1 NP 031488.2 NP_001069454.2
KYRQLLRKHNCLQILLHHLKSSSLTIVSNACGTLWNLSARNKADODLLWELGAVSMLKNL EHROILRENSCLQTLLQHLKSHSLTIVSNACGTLWNLSARNAKDQEALWDMGAVSMLKNL DHROILRENNCLQTLLQHLKSHSLTIVSNACGTLWNLSARNAKDQEGLWDMGAVSMLKNL DHRQILRENSCLQTLLQHLKSHSLTIVSNACGTLWNLSARNAKDQEALWDMGAVSMLKNL DHRQILRENSCLQTLLQHLKSHSLTIVSNACGTLWNLSARNTKDQEALWDMGAVSMLKNL DHROILRENNCLQTLLQHLKSHSLTIVSNACGTLWNLSARNPKDQEALWDMGAVSMLKNL DHROILRENNCLQTLLQHLKSHSLTIVSNACGTLWNLSARNPKDQEALWDMGAVSMLKNL DHROILRENNCLQTLLQHLKSHSLTIVSNACGTLWNLSARNPKDQEALWDMGAVSMLKNI

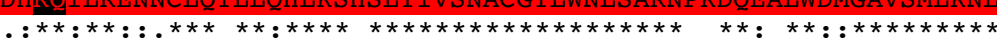

ISSKHKMIAMGSSAALRNLMASRPDVLATADGQK--EGTPGLHVRKQRALQAEIDKN-LK IHSKHKMIAMGSAAALRNLMANRPAKYKDANIMSPGSSLPSLHVRKOKALIEELDAOHLS IHSKHKMIAMGSAAALRNLMANRPAKYKDANIMSPGSSVPSLHVRKOKALEAELDAOHLS IHSKHKMIAMGSAAALRNLMANRPAKYKDTNIMSPGSSLPSLHVRKQKALEAELDAQHLS IHSKHKMIAMGSAAALRNLMANRPAKYKDANIMSPGSSLPSLHVRKOKALEAELDAQHLS IHSKHKMIAMGSAAALRNLMANRPAKYKDANIMSPGSSLPSLHVRKQKALEAELDAQHLS IHSKHKMIAMGSAAALRNLMANRPAKYKDANIMSPGSSLPSLHVRKQKALEAELDAQHLS IHSKHKMIAMGSAAALRNLMANRPAKYKDANIMSPGSSLPSLHVRKQKALEAELDAQHLS IHSKHKMIAMGSAAALRNLMANRPAKYKDANIMSPGSSLPSLHVRKQKALEAELDAQHLS $* * * * * * * * * *: * * * * * * * . * * \quad:: \quad . \quad \ldots * . * * * * *: * * \quad *: *: *$.

DTYAEMEGRTDOHGLLOSOORASLRNRGHRSROOOHGP---DYSPV--------PORIPIW ETFDNIDNLSPKA-----------SHRVKPRHKHNVYGDY--------DAVCRSDGY ETFDNIDNLSPKT------------THRNKORHKONLCSEYALDSSRHDDS ICRSDNF ETFDNIDNLSPKA------_-------SHRNKORHKONIYGEYVLDSSRHDDGVCRTESF ETFDNIDNLSPKT------------SHRPKQRHKQSVYGEYALDASRHDDS--RPDAF ETFDNIDNLSPKA--------------SHRSKORHKONLYGDYAFDANRHDDS--RSDNF ETFDNIDNLSPKA-------------SHRSKQRHKQNLYGDYVFDTNRHDDN--RSDNF ETFDNIDNLSPKA------_------SSRSKORHKOSLYGDYVFDTNRHEDN--RSDNF ETFDNIDNLSPKA--------------SHRSKQRHKQSLYGDYVFDTNRHDDN--RSDNF $: *:::$ : : : **: : : : :*

NPNATPLPDSLMNSQQRAHSPSNRHHGNMINSPETCSDDQSFQEGKTKD-SEGQSTSSQP NPNGVGVRSPYMNTPVLSSPSSRDNRGNAESVRA--ERDRSLDRERRGFLPD-------SIGNLTVLSPYINTTVLPGSS-S-PRPTMDGSRP--EK----DRERTAGLGNYHSTTESS NTGNMTVLSPYLNSTVLPGSA-SSSRGNIENCLS--EKDRSLDRDRAVGLNAYHPATENS STGNLTVLSPYLNSTVLPGSS--SSRTSLESSRS--EKDRSLDRERAVALSTFHPAADSP NTGNMTVLSPYLNTTVLPSSS--SSRGSLDSSRS--EKDRSLERERGIGLSAYHPTTENA NTGNMTVLSPYLNTTVLPSSS--SSRGSLDSSRS--EKDRSLERERGISLGNYHPATENP NAGNMTVLSPYLNTTVLPSSS--SSRGSLDSSRS--EKDRSLERERGIGLGNYHPATENP NTGNMTVLSPYLNTTVLPSSS--SSRGSLDSSRS--EKDRSLERERGIGLGNYHPATENP

$$
\text { - . : . : * } \quad \text { : . . . } \quad . \quad \text { : }
$$

GSVE--NSPGRPHGASRIAQIMOEVAQDGLPTDSS-SGSESPRRESLESRLQSRNGHQOK -GEAAKRMMOIPTSAAQIAVVMEEVQNMHLGMDDRSAGSTPDPHSVQDD--MIRRQ---T GNSSKRIGIQLSTT-AQISKVMDEVSNIHLVQENRSSGSASEMHCMSDERNSQRKP---S GNSSKRIGMOISTAAAOIAKVMEEVTSMH IPQEDRSSGSTSEMHCLTEDRNTTRRA---A GNPSKRLGMQLSTTTAQIAKVMEEVSAIHAQ-EDQSSASTTDLHCVAEERSTLRRA---S GTSSK-RGLQITTTAAQIAKVMEEVSAIHTSQDDRSSASTTEFHCVADDRSAARRS---S GTSSK-RGLQISTTAAQIAKVMEEVSAIHTSQEDRSSGSTTELHCGTDERNALRRS---S GTSSK-RGLOISTTAAOIAKVMEEVSAIHTSOEDRSSGSTTELHCVTDERNALRRS---S GTSSK-RGLQISTTAAQIAKVMEEVSAIHTSQEDRSSGSTTELHCVTDERNALRRS---S

$$
::^{*}::^{*}: * * \quad: . \text { * }^{*} \quad: \quad \text {. * }
$$

PTCPRSSSFTHMPPEGSNLSSRSNSYCFGFDGGHGLVARRSSTESINSISSDIFPAGIHE AVHGHONIYSYSKTDPSG------RPCPM--PKL--EY-RASNDSLNSVNSTD---GYGSNHPQSNPFTFTKAESST------RGCPV--AFMKMEYKMASNDSLNSVSSTE---GYGTAHTHSNTY-FPKSENSS------RPCPV--PYTKMEYKRASNDSLNSVSSSD---GYGAAHAHSNTYNFPKPDNSN------RTCAM--PYAKVEYKRSSNDSLNSVSSSD---GYGASHTHSNTYNFTKSENSN------RTCSM--PYAKVEYKRSSNDSLNSVTSSD---GYGTTHTHANTYNFTKSENSN------RTCPI--PYAKVEYKRSSNDSLNSVSSSD---GYGAAHTHSNTYNFTKSENSN------RTCSM--PYAKLEYKRSSNDSLNSVSSSD---GYGAAHTHSNTYNFTKSENSN------RTCSM--PYAKLEYKRSSNDSLNSVSSSD---GYG-

$$
\text { : . : . : * * :*.:*:**:.* * }
$$

RLAQNRSQMDHSQ---SADSSLNMHGTGRSLQNTTALVHSAD--EAFGTNMDSTTNYSLK ----KRGQMKP SVDSYSEDDEGKCCVYRKYPADLAHK I HNANHMEDDNGDLDTPINYSLK ----KRGQVKPSVESYSEDDESKFF SYGQYPAGLAHKIQSANHMDDNDTELDTPINYSLK ----KRGQMKP S IESYSEDDESKFCSYGQYPADLAHKIHSANHMDDNDGELDTPINYSLK ----KRGQMKPS IESY SEDDESKFCSYGQYPADLAHKIHSANHMDDNDEELDTPINYSLK ----KRGQMKPSVESYSEDDESKFCSYGQYPADLAHKIHSANHMDDNDGELDTPINYSLK 
XP 014996065.1 AA $\bar{A} 03586.1$

$\mathrm{XP}$ 783363.3 NP 001137312.1 NP_001084351.1 $\mathrm{XP}-004949340.1$ $\mathrm{XP}-007497871.1$ NP_031488.2 NP 001069454.2 XP_014996065.1 AAĀ 03586.1

XP_783363.3

NP_001137312.1 NP 001084351.1 XP_004949340.1 $\mathrm{XP}-007497871.1$ NP 031488.2 NP_001069454.2 XP 014996065.1 AĀ̄ 03586.1

$\mathrm{XP}$ 783363.3 NP 001137312.1 NP_001084351.1 XP_004949340.1 XP_007497871.1 NP_031488.2 NP 001069454.2 XP_014996065.1 AA $\bar{A} 03586.1$

XP 783363.3 NP_001137312.1 NP 001084351.1 XP_004949340.1 $\mathrm{XP}-007497871.1$ NP_031488.2 NP_001069454.2 $\mathrm{XP} 014996065.1$ AĀ̄ 03586.1

$\mathrm{XP}$ _783363.3 NP 001137312.1 NP_001084351.1 $\mathrm{XP} 004949340.1$ $\mathrm{XP}-007497871.1$ NP 031488.2 NP 001069454.2 XP_014996065.1 AA $\bar{A} 03586.1$

$\mathrm{XP}$-783363.3 NP 001137312.1 NP_001084351.1 XP_004949340.1 $\mathrm{XP} 007497871.1$ NP 031488.2 NP_001069454.2 $\mathrm{XP} 014996065.1$ AĀ̄ 03586.1

XP 783363.3 NP_001137312.1
-- - KRGQMKPS IESYSEDDESKFCSYGQYPADLAHKIHSANHMDDNDGELDTPINYSLK --- KRGQMKPSIESYSEDDESKFCSYGQYPADLAHKIHSANHMDDNDGELDTPINYSLK :*.*. $*$ * *. : : : . : : : * : : . : :*: ****

1031

1066

1087

1090

1087

1085

1087

1087

1087 YSDEOLNSGROSPSONERWARPKH IFDEIKOSEOROSRNOSTTYPVYTES---TDDKHI **:** * :**.:. :

---LSQPCOKCNSPHRPREGLAIGNSYPASOQORPFPOOYSDOSVSSHHNVID------KYQPRFVQQD-LPAFRSR----GSNEQ---------------ISSGSSHGLNKKISQTIC KFPPHFNQSENVPAYTRSRGANNQVDQ-------------SRVSSNLSNNSKASKPHC KYQSPFGQQDCVPSFRSR-GS-NGSDQ-------------NRVGSTLGINQKVNQSLC KFQSRFGQQECVSPYRSR-GA-SGSEQ-------------NRVSSGHGINQKVNQSLC KFQPHFGQQECVSPYRSR-GT-SGSET------------NRMGSSHAINQNVNQSLC KFQPHFGQQECVSPYRSR-AA-NGSET--------------NRVGSNHGISQNVNQSLC KFOPHFGQOECVSPYRSR-GA-NGSET------------NRVGSNHGINONVSOSLC KFQPHFGQQECVSPYRSR-GA-NGSET------------NRVGSNHGINQNVSQSLC

1081

1106

1132

1133

1130

1128

1130

1130

1130

1117

1159

1192

1191

1188

1186

1187

1187

1187 QEDDYEDDKPTNYSERYSEEEQHEE-EERPTNYSIK-YNEEKHHVDQPIDYSLKYATDIQEDDYEDDKPTNYSERYSEEEQHEE-EERPTNYSIK-YNEEKRHVDQPIDYSLKYATDI-

$$
\text { : *.* : : * ..: : * * } * \text { : : }
$$

1177

1214

1247

1247

1243

1241

1243

1243

1243

PSSOKOSFSFSKSSSGOSTKTEHISSSSENTSTPSSNAKRONOLHPSSAOSRSGOT--PSSQKQSFSFSKSSSGQSSKTEHMSSSSENTSTPSSNAKRQNQLHPSSAQSRSGQP---. : * **. . *

RHSIASSHHEDEEEPPCSHDDNTKTYCVEGTPGPISRCSSLSSLDLNEELDEVEKTKDDS -RA---VQKNPTCKAPTINQETLQTYCVEDTP ICFSRGSSLSSLSSEEDEMESCKRNVNS -R-----PKQIPNKPPS INQETIQTYCVEDTP ICFSRGSSLSSLSSAEDEIEGRERNSRG ------QKTASCKTPS INQETIQTYCVEDTP ICF SRCSSLSSLSSAEDE I-GRDQSTRV ------QKTASCKAPSINQETIQTYCVEDTP ICF SRCSSLSSLSSAEDEI-GRDQTTRG ------QKGTTCKVPSINQETIQTYCVEDTP ICF SRCSSLSSLSSADDE I-GCDQTTQE -PK----ATSSSCKVPS INQETIQTYCVEDTPICF SRCSSLSSLSSAEDEV-GCDQTTQE -QK----A--ATCKVSS INQETIQTYCVEDTP ICF SRCSSLSSLSSAEDEI-GCDQTTQE -QK----A--ATCKVSS INQETIQTYCVEDTPICFSRCSSLSSLSSAEDEI-GCNQTTQE

$$
\text { : : : : : : ****.** : ** } * * * * * . \quad:: \quad \text {. . }
$$

AHSPSNDLQSTTPPNEP--------------TPEKVTN------------------KQVI A-SNYPTLPISEKQSTNN-VAADQRTSESQSSVHYVRAKPPRHHL--GHGDGSRHHKTVE Q-ESNNTLQITEPKEISA-VSKDGAVNETRSSVHHTRTKNNRLQTSNISPSDSSRHKSVE T-DTNATLQISELKENSGALSAEAAVSEITSTSQHIRTKSSRLPTSSLSPSESSRHKAVE S-ETTNTLQIAELKENSASVSTGDTGSEVPSTSQH IRTKANRLQTTSLSPSDSTRHKAVE A-DSANTLQTAEVKENDVTRSAEDPATEVPAVSQNARAKPSRLQASGLSSESTRHNKAVE A-ESANTLQIAEIKDNSGPRSNEDSVSKVPAGSQHIRTKSSRLQASGLSSESARH-KAVE A-DSANTLQIAEIKDKIGTRSTEDPVSEVPAVSQHTRTKSSRLQGSSLSSESTRH-KAVE A-DSANTLQIAEIKEKIGTRSAEDPVSEVPAVSQHPRTKSSRLQGSSLSSESARH-KAVE

1265

1326

1359

1358

1354

1352

1355

1353

1353

----------LQLPSDVHHHDEVQETPLVFSRCSSVCSLSSDDVPDICDDVSSIYTNS FSSGAKSPSKSGAQTP-KSPPEHYVQETPLMFSRCTSVSSLDSFESHSIASSVQSEP-CS
1313

1384 
NP 001084351.1 XP_004949340.1 XP_007497871.1 NP 031488.2 NP_001069454.2 XP 014996065.1 AAĀ 03586.1

XP_783363.3 NP 001137312.1 NP_001084351.1 XP_004949340.1 XP 007497871.1 NP_031488.2 NP 001069454.2 XP_014996065.1 AAĀ 03586.1

XP 783363.3 NP_001137312.1 NP 001084351.1 XP_004949340.1 XP_007497871.1 NP 031488.2 NP_001069454.2 XP 014996065.1 AAĀ 03586.1

XP_783363.3 NP 001137312.1 NP_001084351.1 XP 004949340.1 XP 007497871.1 NP 031488.2 NP 001069454.2 XP_014996065.1 AAĀ 03586.1

XP_783363.3 NP_001137312.1 NP 001084351.1 XP_004949340.1 XP 007497871.1 NP 031488.2 NP_001069454.2 XP 014996065.1 AAĀ 03586.1

XP 783363.3 NP 001137312.1 NP_001084351.1 XP 004949340.1 XP_007497871.1 NP 031488.2

NP 001069454.2 XP 014996065.1 AAĀ03586.1

XP 783363.3 NP_001137312.1 NP 001084351.1 XP 004949340.1 XP_007497871.1 NP 031488.2 NP_001069454.2 XP 014996065.1 AAA03586.1
FSSGAKSPSKSGAQTP-KSPPEHYVQETPLMFSRCTSGSSLDSFESHSIASS IASSVASE FSSGAKSPSKSGAOTP-KSPPEHYVOETPLMFSRCTSVSSLDSFESRSIASSVOSEP-CS FSSGAKSPAKSGAQTP-KSPPEHYVQETPLMFSRCTSVSSLDSFESRSIASSVQSEP-CS FSSGAKSPSKSGAOTP-KSPPEHYVQETPLVFSRCTSVSSLDSFESRSIASSVQSEP-CS FSSGAKSPSKSGAQTP-KSPPEHYVQETPLMFSRCTSVSSLDSFESRSIASSVQSEP-CS FSSGAKSPSKSGAOTP-KSPPEHYVOETPLMFSRCTSVSSLDSFESRSIASSVQSEP-CS FSSGAKSPSKSGAQTP-KSPPEHYVQETPLMFSRCTSVSSLDSFESRSIASSVQSEP-CS

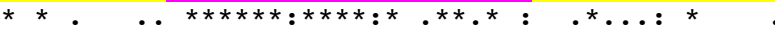

RAASGFVSPSELPDSPSDTMPPSPRRTSQS---------NVKVDL-------------GMVSGIISPSDLPDSPGOTMPPSRSKTPPPPPPRSTS---VKOKV--TVPPHTEKHDLAP HMISGIISPSDLPDSPGQTMPPSRSKTPP-----PPQTVQAKKDGSKPIVPDEERG--KV GMVSGI ISPSDLPDSPGQTMPPSRSKTPP-----PAQGVQVKRDVTKGKVPTAEKREPGP GMVSGIISPSDLPDSPGOTMPPSRSKTPP-----PPOTGOTKREATKSKLPSAEKRESGP GMVSGI ISPSDLPDSPGQTMPPSRSKTPP----PPPQTVQAKREVPKSKVPAAEKRESGP GMVSGI ISPSDLPDSPGQTMPPSRSKTPPPPPPPPPQTVQTKQEVPKNKAPSAEKRESGP GIVSGI ISPSDLPDSPGQTMPPSRSKTPPP----PPQTAQTKREVPKNKTPTAEKRESGP GMVSGIISPSDLPDSPGQTMPPSRSKTPPP----PPQTAQTKREVPKNKAPTAEKRESGP

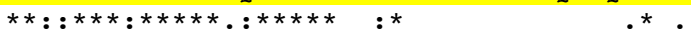

- -LNAPKLPEMEEKPTMYATEGTPIDNSCATSLSALTIDGDVK-ATVAKRLLP RHAVVSAAVQKVQVLPD-NDTLLHFATESTPDGF SCASSLSALSLDEPF IQKDVELKIMP AKTAVHSAIORVOVLOE-ADTLLHFATESTPDGF SCASSLSALSLDEPYIOKDVOLKIMP RQAAVNAAVQRVQVLPD-ADTLLHFATESTPDGF SCSSSLSALSLDEPF IQKDVELRIMP RQAAVNAAVORVOVLPD-ADTLLHFATESTPDGF SCSSSLSALSLDEPF IOKDVELRLMP KOTAVNAAVQRVOVLPD-VDTLLHFATESTPDGFSCSSSLSALSLDEPF IOKDVELRIMP KQAAVNAAVQRVQVLPE-ADTLLHFATESTPDGF SCSSSLSALSLDEPF IQKDVELRIMP KOAAVNAAVQRVOVLPD-ADTLLHFATESTPDGFSCSSSLSALSLDEPFIOKDVELRIMP KQAAVNAAVQRVQVLPD-ADTLLHFATESTPDGF SCSSSLSALSLDEPF IQKDVELRIMP

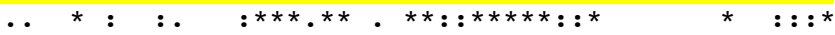

1418

1416

1412

1410

1413

1411

1411

1349

1439

1471

1471

1467

1466

1473

1467

1467

1399

1498

1530

1530

1526

1525

1532

1526

1526

PKGNEGSENVGSEDKE-----ITDEKNVEPS-------GNEFSEQEQSLLDECINAAMPV PVHEDDHSIEAEPEMEDMHEPKVOEKSPATSEAAKDILDDSDDDDDTEILNACINSAMPT PVLENDQGNKAEPEKE--F IDNKAKKEDKRSEQEKDMLDDT--DDDIDILEECIISAMPR PVHENEHGNEAEPEOSDETKDNOENKAEKPSEAEKDILDDSD-DDDIEILEECIISAMPT PVQENEHGNETEPEQLEDVSEVKEKKAEKPTESEKDILDDSD-DDDIEILEECI ISAMPT PVQENDNGNETESEQPEESNENQDKEVEKP-DSEKDLLDDSD-DDDIEILEECIISAMPT PVOENDNGNETESEOPEESNENOEKEAEKPTDSEKDLLDESD-DDDIEILEECI ISAMPT PVQENDNGNETESEQPKESNENQEKEAEKTIDSEKDLLDDSD-DDDIEILEECI ISAMPT PVQENDNGNETESEQPKESNENQEKEAEKTIDSEKDLLDDSD-DDDIEILEECI ISAMPT

$$
\text { * : : } \quad .: \quad:: \quad .: \quad::::^{*}: * *: * * *
$$

1447

1558

1586

1589

1585

1583

1591

1585

1585

RRKGIPKPT----------KSKSKREITSRKIPVSHKPSSSKHVSFEKSNAAPPTTMO KSSRKPKKQ---STSRIPPPVACKP SQLPVYKLLPPQNRGQPQKHVALA--------HGE KPSRKNKKVPQPTPGKPPPPVARKPSOLPVYKLLSSQNRLQTQKHVNFT---------HSD KSSRKAKKPSQASAPKIPPPVARKPSQLPVYKLLPSQSRLQSQKHVSFT---------PGD KSSRKAKKPSOA-ASKIPPPVARKPSOLPVYKLLPSONRLOAOKHVSFT---------PGD KSSRKAKKLAQT-ASKLPPPVARKPSQLPVYKLLPAQNRLQAQKHVSFT---------PGD KSSRKAKKPAQT-TSKLPPPVARKPSQLPVYKLLPSQNRLQAQKHVSFT--------PGD KSSRKAKKPAOT-ASKLPPPVARKPSOLPVYKLLPSONRLQPOKHVSFT--------PGD KSSRKAKKPAQT-ASKLPPPVARKPSQLPVYKLLPSQNRLQPQKHVSFT--------PGD : . $*$ *..$\quad$ : : . : . .*** :

1495

1607

1638

1641

1636

1634

1642

1636

1636

DLPKPFCTEDTPVNFSAATSLSDLSIDDIEIESSSDGKLMIANPDRSALEKPSGIESDNO DMPRVYCVEGTPINFSTATSLSDLTIDSPPNELAGMESSA-PHVEA-SGQRRDTL----DMPRVYCVEGTPINF STATSLSDLTIESPPSEPTND-QPN-TDSLSTDLEKRDTI----DMPRVYCVEGTPINFSTATSLSDLTIESPPNELANVDSVG-AGAESGEFEKRDTI----DVPRVYCVEGTPINFSTATSLSDLTIESPPNELAGVEGTS-TGALLGDFEKRDTI----DVPRVYCVEGTPINFSTATSLSDLTIESPPNELATGDGVR-AGIQSGEFEKRDTI-_--DMPRVYCVEGTPINFSTATSLSDLTIESPPNELAAGEGVR-AGAQSSEFEKRDTI----DMPRVYCVEGTPINFSTATSLSDLTIESPPNELAAGEGVR-AGAQSGEFEKRDTI----DMPRVYCVEGTPINFSTATSLSDLTIESPPNELAAGEGVR-GGAQSGEFEKRDTI-----

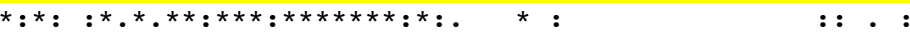
VRTSGDLLHCMDDAR-----SETNSVTKEAEDSMLOECINSAIPRSKSCKPRSSLLAKHR -PE-GKSAEAKETGLSPPM-QSA--LAENEGDDILAECINSAMPKSKIHKPFRVQKMP--PTEGRSTDDTDASKPLNP-TTVLDEDKAEEGDILAECIHSAMPKGKSHKPYRVKKIM--PTEGRSTDDTORAKSITVTGPGLDDDKTEEGDILAECINSAMPKGKSHKPFRVKKIM--PTEGRSTDDIQIGKSSNVNTSAFDDNKTEEGEILAECINSAMPKGKSHKPFRVKKIM--PTEGRSTDDAORGKISSIVTPDLDDNKAEEGDILAECINSAMPKGKSHKPFRVKKIM--PTEGRSTDEAQRGKASSVTVPELDDSKTEEGDILAECINSAMPKGKSHKPFRVKKIM--PTEGRSTDEAQGGKTSSVTIPELDDNKAEEGDILAECINSAMPKGKSHKPFRVKKIM--PTEGRSTDEAQGGKTSSVTIPELDDNKAEEGDILAECINSAMPKGKSHKPFRVKKIM-: $\quad$ : :* ***:**: : * ** 
XP_783363.3

NP_001137312.1 NP_001084351.1 XP_004949340.1 $\mathrm{XP} 007497871.1$ NP_031488.2 NP_001069454.2 XP 014996065.1 $\mathrm{AA} \overline{\mathrm{A}} 03586.1$

XP_783363.3 NP 001137312.1 NP_001084351.1 XP 004949340.1 XP_007497871.1 NP 031488.2

NP_001069454.2 XP_014996065.1 AAA 03586.1

XP_783363.3 NP_001137312.1 NP 001084351.1 XP_004949340.1 XP 007497871.1 NP_031488.2 NP_001069454.2 $\mathrm{XP}-014996065.1$ $\mathrm{AA} \overline{\mathrm{A}} 03586.1$

XP 783363.3 NP_001137312.1 NP_001084351.1 XP 004949340.1 XP_007497871.1 NP 031488.2 NP_001069454.2 XP_014996065.1 AAA 03586.1

XP_783363.3 NP 001137312.1 NP 001084351.1 XP 004949340.1 XP 007497871.1 NP_031488.2 NP 001069454.2 XP_014996065.1 AA $\bar{A} 03586.1$

XP 783363.3

NP_001137312.1 NP 001084351.1 $\mathrm{XP} 004949340.1$ $\mathrm{XP} 007497871.1$ NP 031488.2 NP_001069454.2 XP 014996065.1 AAĀ 03586.1

XP_783363.3 NP 001137312.1 NP_001084351.1 XP 004949340.1 XP_007497871.1 NP_031488.2
SPROIGKFRSPKGVAPRKRLPSADVRHKSPSPIPEMSSDTRSTSSQERGQDWGNNPSPPP --DQAQHPSTATGS---LV-QQDLEKKKPTSPVKPMPQSSEYRARMLKRPEANNS----L --DOINHTSAATSSGNSRS-MOETDKNKPTSPVKPMPOSIGFKERLKKNTELKLN----P --DQIQQASTS---LNNKN-QPEGEKKKPTSPVKPVPQNSEYRARVRKNTESKSQ----I --DQIOQASAS-SSGNSKN-PLDSEKKKPTSPVKPMPOSAEYRTRIRKNAESK-N----V --DQVQQASST-SSGANKN-QVDTKKKKPTSPVKPMPQNTEYRTRVRKNTDSKVN----V --DQVQQASMS-SSGTNKN-QLDGKTKKPTSPVKPIPQNTEYRTRVRKNTDSKNN----I --DQVQQASAS-SSATNKN-QLDGKKKKPTSPVKPIPQNTEYRTRIRKNADSKNN----I --DQVQQASAS-SSAPNKN-QLDGKKKKPTSPVKPIPQNTEYRTRVRKNADSKNN----L

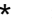
$: * \quad *$ : :

DTVTTYCMEGTPGISNATSLSDLTSPIDSEDVNGNITSONLPONRSTLGVGVSRGSTPSD ADPATYPDKNKETR-------KOEP-----KVVIRDFADKPSNAE-----ERTRPGFAFD NSENQYCDP-R---------KPSS-----KKPSKVANEKIPNNE-----ERTKG-FAFD NNERSYPEN-RDAK-------KONL-----KNNSRDFNDKLPNNE------ERVRGSFTFD NVERSYS-D-KDSK-------KLSL-----KNNARDFLDKMPNNE-----DRVRGSFTFD NTEETF SDN-KDSK-------KPSL-----OTNAKAFNEKLPNNE-----DRVRGSFALD NAERNFSEN-KDSK-------KQHL-----KNNSKDFNDKLPNNE-----DRVRGSFTFD NAERVF SDN-KDSK-------KQNL-----KNNSKDFNDKLPNNE-----DRVRGSFAFD NAERVFSDN-KDSK-------KQNL-----KNNSKDFNDKLPNNE-----DRVRGSFAFD

$$
\text { : } \quad \text { • } \quad \text {. } \quad \text { : : } \quad: \text {. }
$$

1670

1763

1800

1802

1798

1797

1805

1799

1799

1730

1806

1838

1844

1839

1839

1847

1841

1841

TPR-VFAVEGTPINFSCNGSLSSLSCDEDA-ELTEAKAQMKEVSKRNQAGGGMSRQQTVI SPHHYTPIEGTPYCF SRNDSLSSLDFEDEDLDFSKEKAVLRKDKEORKVPL------LKSPHHYTPIEGTPYCF SRNDSLSSLDFEDDDIDLSKEKAELRKEKGTKDTDO-------KVSPHHYTPIEGTPYCF SRNDSLSSLDFDDDDVDLSREKAELRKGKEAKEVET-------KDSPHHYTPIEGTPYCFSRNDSLSSLDFDDDDVDLSREKAEL-KGKEAKETEA------KVSPHHYTPIEGTPYCF SRNDSLSSLDFDDDDVDLSREKAELRKGKESKDSEA------KVSPHHYTPIEGTPYCFSRNDSLSSLDFDDDDVDLSREKAELRKGKENKESEA------KVSPHHYTPIEGTPYCF SRNDSLSSLDFDDDDVDLSREKAELRKAKENKESEA-------KVSPHHYTPIEGTPYCF SRNDSLSSLDFDDDDVDLSREKAELRKAKENKESEA------KV-

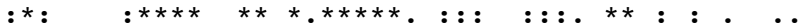

PKORASFSNPIEOVOSPENKYYSMDDYHNAEASKSAMSPLDSPRVFAVEGTPGIISRADS ------CS-VEQ---PANTNM--------VSTFQT---------APTKP--L-----------CPNVEQ---PSGQQP---------SNRTQV----------COKHP--TSRSQ-------SNHLEL---TSNQQS---------ANRAQI----------CAKHP--VERGQ-------TCRPEP---NSSOOA--------ASKSOA---------SIKHP--ANRAO-------TNHTEL---TSNQQS--------ASKTPA---------VTKQP--INRGQ-------TSHTEL---TSNQQS--------ASKTQA---------IAKHP--INRGQ------TSHTEL---TSNQQS--------ANKTQA---------IAKQP--INRGQ-

LSSLSCDEEDASPAVEKSAKERLAEKTRTSRISGSRMNHLGEGSMAMRRLSSEEAPLSYA ----------------QK--------T-V--F------PQAPKEN---TVVVCDEKQKFS ---------PKALVQKP--------T-S--F------SSAAKGTQDRGGATDEKMENFA

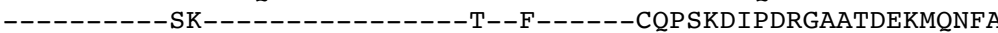
---------SKPLLQKQ--------S-T--F------PQSSKDMPDRVAATDEKLQNFA ----------SKPVLQKQ--------P-T--F------PQSSKDGPDRGAATDEKLQNFA ----------SKPVLQKQ--------S-T--F------PQSSKDIPDRGAATDEKLQNFA ---------LKPILQKQ--------S-T--F------PQSSKDIPDRGAATDEKLHNFA -------- PKP ILQKQ--------S-T--F------PQSSKDIPDRGAATDEKLQNF $: \quad .:$ :

VEDTPTCFSHNSSLSALSDNDEEERPDLTEOW-HDDEESOPORSSGGANGINKDSRRVFA IEDTPVCFSRNSSLSSLSDIDQENNNKDCSHK-----DDVTQ-MEAPRPQASGYAPKAFH IENTPVCFSRNSSLSSLSDIDQENN-NKETEPLKQTGTSETQ-LGLRRPQTSGYAPKSFH IENTPVCFSRNSSLSSLSDIDQENNNNKEGEPVKRTEAPDSQ-IESSRPQTSGYAPKSFH IENTPVCF SRNSSLSSLSDIDQENNNNKESEPTKETEPPDSQ-GEPNRPQTSGYAPKSFH IENTPVCFSRNSSLSSLSDIDQENNNNKESEPIKEAEPANSQ-GEPSKPQASGYAPKSFH IENTPVCFSRNSSLSSLSDIDQENNNNKENEPVKETEPPASQ-GEPGKPQASGYAPKSFH IENTPVCFSHNSSLSSLSDIDQENNNNKENEPIKETEPPDSQ-GEPSKPQASGYAPKSFH IENTPVCF SHNSSLSSLSDIDQENN-NKENEPIKETEPPDSQ-GEPSKPQASGYAPKSFH

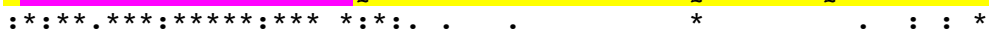

1788 1859

1891

1897

1891

1892

1900

1894

1894

1848

1882

1919

1925

1919

1920

1928

1922

1922

1908

1906

1952

1951

1952

1953

1961

1955

1955

1967

1960

2010

2010

2011

2012

2020

2014

2013

TEDTPVCFSRNSSLSSLDAESDGDAASEQALLDECITSGMPQSKVKKPVKRI---_----VEDTPVCFSRNSSLSSLSIDS------EDDLLQECISSAMPKKKKOTPRSKTEESGVKEE VEDTPVCF SRNSSLSSLSIDS------EDDLLQECISSAMPKKRKPSKIKNE----VGKS VEDTPVCFSRNSSLSSLSIDS------EDDLLQECISSAMPKKKKPSRIKSE----SEKS VEDTPVCF SRNSSLSSLSIDS------EDDLLOECISSAMPKKKRPSRFKGD----DEKP VEDTPVCF SRNSSLSSLS IDS------EDDLLQECISSAMPKKKRPSRLKSE----SEKQ
2019 2014 2060

2060

2061

2062 
NP 001069454.2 $\mathrm{XP}-014996065.1$ AĀ̄ 03586.1

$\mathrm{XP}$-783363.3

NP_001137312.1 NP 001084351.1 $\mathrm{XP}-004949340.1$ XP_007497871.1 NP 031488.2

NP 001069454.2 $\mathrm{XP} 014996065.1$ $\mathrm{AA} \overline{\mathrm{A}} 03586.1$

$X P \_783363.3$ NP 001137312.1 NP 001084351.1 $\mathrm{XP}-004949340.1$ $\mathrm{XP}-007497871.1$ NP_031488.2 NP 001069454.2 XP_014996065.1 AA $\bar{A} 03586.1$

XP_783363.3 NP_001137312.1 NP 001084351.1 XP_004949340.1 $\mathrm{XP}-007497871.1$ NP 031488.2 NP_001069454.2 XP_014996065.1 AĀ̄ 03586.1

$\mathrm{XP}$ 783363.3 NP 001137312.1 NP_001084351.1 XP_004949340.1 $\mathrm{XP}-007497871.1$ NP 031488.2 NP 001069454.2 XP_014996065.1 AĀ̄03586. 1

XP 783363.3 NP_001137312.1 NP 001084351.1 XP_004949340.1 XP_007497871.1 NP 031488.2 NP_001069454.2 XP 014996065.1 AA $\bar{A} 03586.1$

XP 783363.3 NP 001137312.1 NP_001084351.1 $\mathrm{XP}-004949340.1$ XP_007497871.1 NP 031488.2 NP 001069454.2 XP_014996065.1 AA $\bar{A} 03586.1$

$\mathrm{XP}$ 783363.3 NP_001137312.1 NP_001084351.1
VEDTPVCFSRNSSLSSLSIDS------EDDLLQECISSAMPKKKKPSRLKPD----NEKH VEDTPVCFSRNSSLSSLSIDS------EDDLLQECISSAMPKKKKPSRLKGD----NEKH VEDTPVCFSRNSSLSSLSIDS------EDDLLQECISSAMPKKKKPSRLKGD----NEKH .****************.:* $:$ : $* *: * * *: * * *: .:$.

$----N G K I I S G G P S S M-$

KSMMADGILSEEPDLILDLTDTHSPISEQALSPDSESFDWKAIQEGANSIVSSLHQAA-RSNSVGGILAEEPDLTLDLRDIQSPDSENAF SPDSENFDWKAIQEGANSIVSRLHQAA-A NSRNIGGMLAE--DLTLDLREIORPDSEHGF SPDSENFDWKAIOEGANS IVSSLHOAA-A SPRNMDGILAE--DLTLDLRDIQRPDSEHGF SPDSENFDWKAIQEGANSIVSSLHQAA-A SPRKVGGILAE--DLTLDLKDLORPDSEHAF SPDSENFDWKAIOEGANS IVSSLHOAAAA SPRNMGGILAE--DLTLDLKDIQRPDSEHGLSPDSENFDWKAIQEGANSIVSSLHQAA-A SPRNMGGMLAE--DLTLDLKDIQRPDSEHGLSPDSENFDWKAIQEGANSIVSSLHQAA-A SPRNMGGILGE--DLTLDLKDIQRPDSEHGLSPDSENFDWKAIQEGANSIVSSLHQAA-A . : : .

-ASLSRQGSSDSDSILSLKSGISIGSPFHLPLNQDDKPA-PNKGPRILKPGEKSSIEAKK AGSLSRQGSSDSDS ILSLKSGISLGSPFHLTLDKEEKTITSNKGPKILKPAEKSALENKK AASLSRQASSDSDSILSLKSGISLGSPFHLTPDQEEKPFTSNKGPRILKPGEKSTLESKK AACLSROASSDSDSILSLKSGISLGSPFHLTPDOEEKPFTSNKGPRILKPGEKSTLETKK AACLSRQASSDSDSILSLKSGISLGSPFHLTPDQEEKPFTSNKGPRILKPGEKSTLEAKK AACLSROASSDSDSILSLKSGISLGSPFHLTPDOEEKPFTSNKGPRILKPGEKSTLETKK AACLSRQASSDSDSILSLKSGISLGSPFHLTPDQEEKPFTSNKGPRILKPGEKSTLETKK AACLSRQASSDSDSILSLKSGISLGSPFHLTPDQEEKPFTSNKGPRILKPGEKSTLETKK $::::: \quad . * *$ : $* *$. . : :

EEEEGPKGVKGGKKIYRSPITGKIRSITPPKSVLPPKSPSSTRGGLAKGSPTTSRGRGAI KEEETAKSLKGGKKVYKSLITGKPRPSLES--MAS--------QHRQAQAPVISRGRTMV TEE-EPKGIKGGKKVYKSLITGKSRSSSDFSSHCK--------QSVQTNMPSISRGRTMI VES-ESKGIKGGKRVYKSIITGKARSNSEVSSQIK--------QPQQTSVPSISRGRTMI IES-ENKGIKGGKKVYKSLITGKVRSNSEVSGQLK--------QPLPTNMPSISRGRTMI IES-ENKGIKGGKKVYKSLITGKIRSNSEISSQMK-------QPLPTNMPSISRGRTMI IES-ENKGIKGGKKVYKSLITGKVRSNSEISSQMK--------QPLQTNMPS ISRGRTMI IES-ESKGIKGGKKVYKSLITGKVRSNSEISGQMK--------QPLQANMPS ISRGRTMI IES-ESKGIKGGKKVYKSLITGKVRSNSEISGQMK--------QPLQANMPS ISRGRTMI *. $.: * * * *::$ : * **********

RGARGGFARSASTTPPRSSTPTGRGTPPRTTTPPRTTSPMTAGRTTTPPRTTSPRTTSPR HVP--GVRSSSPSTSPV-----PKKPPPRG-------QMSKPPSQAPGAGSSPRTMKVP HIP--GVRASSPSTSPV-----SKKGPVFKN-------VPSKGSNENPSSSSSPKGTKPI HIP--GVRNSSSSTSPV-----SKKGPPFKN-------TNSKSPSEGQSSASSPRGVKSS HIP--GIRNSSSSTSPV-----SKKGPSLKT-------PTSKSPSEGPTSSTSPRGAKPS HIP--GLRNSSSSTSPV-----SKKGPPLKT-------PASKSPSEGPGATTSPRGTKPA HIP--GVRNSSSSTSPV-----SKKGPPLKT-------PASKSPSEGQPATTSPRGTKPS HIP--GVRNSSSSTSPV-----SKKGPPLKT-------PASKSPSEGQTATTSPRGAKPS HIP--GVRNSSSSTSPV-----SKKGPPLKT-------PASKSPSEGQTATTSPRGAKPS : *. $*$ : : * : * $\quad$ : . $\quad$ : * : .

SGTPPKPKGSIA---NKSITPVRNATNGARS ITPPRPVRKSSVDSQKDGDAKSETSSR-PSSEPSPASG--PPSSQGGSSKASSRSGSRDSTPSRPVQQSLTRPMQSPGRASVSPGRNG KS-ELVY--GSRPSSTPGGSSKGNSRSGSRDSASSRPSPQPLSRPLQSPGRNSISPGKNG VKPEPAPVTRQLSGLNQGGSSKGPSRSGSRDSTPSRPQQQPLSRPLQSPGRNSISPGRNG VKSELSPVTRQTS--QPGGSSKGPSRSGSRDSTPSRPSQQPLSRPMQSPGRNS ISPGRNG GKSELSPITRQTS--QISGSNKGSSRSGSRDSTPSRPTQQPLSRPMQSPGRNSISPGRNG VKSELSPVTRQAS--QTAGSNKGPSRSGSRDSTPSRPAQQPLSRPMQSPGRNS ISPGRNG VKSELSPVAROTS--OIGGSSKAPSRSGSRDSTPSRPAQOPLSRPIOSPGRNS ISPGRNG VKSELSPVARQTS--QIGGSSKAPSRSGSRDSTPSRPAQQPLSRPIQSPGRNS ISPGRNG . : : .*:. : ** : : . * : . :

\begin{tabular}{|c|c|}
\hline RSSKESISSIP-K & 1 \\
\hline LSPSNKLSQLPQLPRTASPSASSTTKSS-----------GS----GRMAY-----TSPGRQ & 23 \\
\hline ISPPNKFSQ---LPRTTSPSTASTKSS-----------GS----GRMSY-----TSPGRQ & 2370 \\
\hline ISPPNKLSQ---LPRTSSPSTASTKSS-----------SS----GRMSY-----TSPGRQ & 2371 \\
\hline ISPPNKLSQ---LPRTSSPSTSSTKSS-----------GS----GKISY-----TSPGRQ & 2370 \\
\hline ISPPNKLSQ---LPRTSSPSTASTKSS-----------GS----GKMSY-----TSPGRQ & 2372 \\
\hline ISPPNKLSQ---LPRTSSPSTASTKSS-----------GS----GKMSY-----TSPGRQ & 2379 \\
\hline ISPPNKLSQ---LPRTSSPSTASTKSS-----------GS----GKMSY-----TSPGRQ & 2373 \\
\hline ISPPNKLSQ---LPRTSSPSTASTKSS-----------GS----GKMSY-----TSPGRQ & 2372 \\
\hline$* * \quad . * .:: * *: *$ & \\
\hline MPVPSRSNSPSTKSSGSVTTPRKPLNKSPSSTKARDES-PTDAEEVTSQMKSMHLESN & 2360 \\
\hline LVQPTP-----TKQSGLPR-STSGIPRSESASKILNQC----GPSKKAELSRM-SSTKSS & 2372 \\
\hline LSQPNL-----SKQSGLPK-THSSIPRSESASKSLNQNVNT-GSN & 2422 \\
\hline
\end{tabular}

2242 2283 2333 2334 2333 2335 2342 2336 2335 
XP 004949340.1 XP_007497871.1 NP_031488.2 NP 001069454.2 XP_014996065.1 AAĀ 03586.1

XP 783363.3 NP_001137312.1 NP 001084351.1 XP_004949340.1 XP_007497871.1 NP 031488.2 NP_001069454.2 XP 014996065.1 AAA $\bar{A} 03586.1$

XP_783363.3 NP 001137312.1 NP_001084351.1 XP 004949340.1 XP 007497871.1 NP 031488.2 NP 001069454.2 XP_014996065.1 AAA 03586.1

XP_783363.3 NP_001137312.1 NP 001084351.1 XP_004949340.1 $\mathrm{XP}-007497871.1$ NP 031488.2 NP_001069454.2 XP 014996065.1 AA $\bar{A} 03586.1$

XP 783363.3 NP_001137312.1 NP_001084351.1 XP 004949340.1 XP_007497871.1 NP 031488.2 NP 001069454.2 $\mathrm{XP} 014996065.1$ AAA03586.1

XP_783363.3 NP_001137312.1 NP 001084351.1 XP_004949340.1 $\mathrm{XP}-007497871.1$ NP 031488.2 NP_001069454.2 XP 014996065.1 $\mathrm{AA} \overline{\mathrm{A}} 03586.1$

XP 783363.3 NP_001137312.1 NP_001084351.1 XP 004949340.1 XP_007497871.1 NP 031488.2 NP_001069454.2 XP_014996065.1 AAA03586.1
MSQQNL-----TKQTALTK-NTSS IPRSESASKGLNQILGSGASNKKTDLSRM-SSAKSS MSOONL-----TKOTGLSK-NTSNIPRSESASKGLNOISNSNGTNKKVELSRM-SSTKSS LSQQNL-----TKQASLSK-NASS IPRSESASKGLNQMSNGNGSNKKVELSRM-SSTKSS MSQQNL-----TKQTGLSK-NGSGIPRSESASKGLNQMSNSNGSNKKVELSRM-SSTKSS MSQQNL-----TKQTGLSK-NASS IPRSESASKGLNQVNNGNGANKKVELSRM-SSTKSS MSQQNL-----TKQTGLSK-NASS IPRSESASKGLNQMNNGNGANKKVELSRM-SSTKSS

: . :*... . : : **: :* : : ... : :.* . : :.*

SPDLDDGDRPVLLKOSTFTKDSASLPEQTP-----EMNPVSGD---------EKVLSPVQS GSESDRSEKPGLVRQSTF IKEAPSPTLKRKLEESASFESLSPSST------SOSQTPVSS GSESDRSERPALVROSTFIKEAPSPTLRRKLEESASFESLSSSSRADSPPRSOTOTPALS GSESDRSERPVLVRQSTF IKEAPSPTLRRKLEESASFESLSP-SRPDSPTRSQLQTPVLS GSESDRSERPVLVRQSTF IKETPSPTLRRKLEESASLESLSSSSRPDSPTKSQVQTPILS GSESDRSERPALVROSTFIKEAPSPTLRRKLEESASFESLSPSSRPDSPTRSOAOTPVLS GSESDRSERPVLVRQSTFIKEAPSPTLRRKLEESASFESLSPSSRPDSPTRSQAHTPVLS GSESDRSERPVLVRQSTF IKEAPSPTLRRKLEESASFESLSPSSRPASPTRSQAQTPVLS GSESDRSERPVLVRQSTF IKEAPSPTLRRKLEESASFESLSPSSRPASPTRSQAQTPVLS . : * : : * *::****::* : $\quad .::$ :*

EEAPSVTESESSVSLSKGGWKKTGSGIOGSOESKKSSTGN-KPVSK--TTGVSKITPRRT PSLPDMSL-SLP--YQGSGWTKAPQSON-SAENGDGKSLKRHDISRSHSESPSRLPINRT PSLPDMAL-STHS-IQAGGWRKMPPNLNPAAEH--GDSRRRHDISRSHSESPSRLPITRS PSLPDMSL-STHSTAOTSGWRKLPPNLSPSVEY-DGRPAKRHDIARSHSESPSRLPINRS PSLPDMSL-STHSSIQTGSWRKLPPNLNPS IEFNDGRSTKRHDIARSHSESPSRLPVNRS PSLPDMSL-STHPSVOAGGWRKLPPNLSPTIEYNDGRPTKRHDIARSHSESPSRLPINRA PSLPDMSL-STHSSLOSGGWRKLPPNLSPTIEYNDGRPVKRHDIARSHSESPSRLPINRS PSLPDMSL-STHSSVQAGGWRKLPPNLSPTIEYNDGRPAKRHDIARSHSESPSRLPINRS PSLPDMSL-STHSSVQAGGWRKLPPNLSPTIEYNDGRPAKRHDIARSHSESPSRLPINRS

$$
\text { . * : : . . . .** . . : * . . : : : : : . * }{ }^{*}::^{*}:
$$

GSPGLRTPAARSSSPGORVNTPARRSESPSRMSSTSORSESPSRASTTSOSSVSKORTTP GTWK--REHSKHSSSLPRVGTWKRTGSSSS ILSASSESSEKG---RS----EDERQP-TN GTWK--REHSKHSSSLPRVSTWRRTGSSSS ILSASSESSEKA---KS----EDEKOO-VC GTWK--REHSKHSSSLPRVSTWRRTGSSSS ILSASSESSEKA---KS----EDEKOH-GS GTWK--REHSKHSSSLPRVSTWRRTGSSSSIISASSESSEKA---KS----EDEKHV--S GTWK--REHSKHSSSLPRVSTWRRTGSSSS ILSASSESSEKA---KS----EDERHV--S GTWK--REHSKHSSSLPRVSTWRRTGSSSSILSASSESSEKA---KS----EDEKOV--N GTWK--REHSKHSSSLPRVSTWRRTGSSSSILSASSESSEKA---KS----EDEKHV--N GTWK--REHSKHSSSLPRVSTWRRTGSSSSILSASSESSEKA---KS----EDEKHV--N *: : : ** **.* *..** :*::* $* * . \quad$ : . .: :

QTKGPASTORNNSATSKINTGLOKSGGMSKRSASPSGVKTNPNVNGTHASTPPKSTASRP

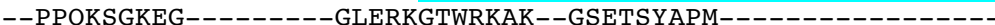
SFPGPRS--EC---------SSSAKGTWRKIK--ESEILETPSNGSSST---------SLSGQKQSKES---------QAPAKGTWRKIK--ENEIPQIMNDPQ-HP----------SLLGTKQTKEN---------QGPAKGTWRKIK--ESEMTPISNVSQ-VT----------SMPAPRQMKEN---------QVPTKGTWRKIK--ESDISPTGMASQ-SA----------SISGSKQTKEN---------QVSTKGTWRKIK--ESEISPTNSTSQ-TT--_--_----SISGTKQSKEN---------QVSAKGTWRKIK--ENEISPTNSTSQ-TV----------SISGTKOSKEN---------OVSAKGTWRKIK--ENEFSPTNSTSO-TV---_--_----

$$
\text { * * . . }
$$

NTRPTKSGNAGVRTGPPTTRVASASPKPSGASARAVRNKPASRPSSAEGSRSLSGNNSSS -----------SLDLQDQTGDAMSKSEDVWV-RIEDCPIN-------------NPRS ----IAESNCSLESKTLVYOMAPAVSKTEDVWV-RIEDCPIN--------------NPRS ----SSSATSSSDSKTLIYQMAPAVSKTEDVWV-RIEDCPIN--------------NPRS ----SSGTTNGADSKTLIYOMAPAVSKTEDVWV-RIEDCPIN--------------NPRS ----SSGAASGAESKPLIYQMAPPVSKTEDVWV-RIEDCPIN-------------NPRS ----SSGAANGAESKTLIYOMAPAVSKTEDVWV-RIEDCPIN-------------NPRS ----SSGATNGAESKTLIYQMAPAVSKTEDVWV-RIEDCPIN-------------NPRS ----SSGATNGAESKTLIYQMAPAVSKTEDVWV-RIEDCPIN-------------NPRS $\therefore \quad$ * $\quad \therefore$ * * * *

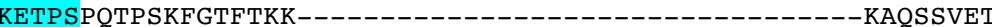
SKSPTASTPPVIDSLPIKGLACDRDSSESHSKLMSENA---AMSHLGSETNLNLLRSSES GRSPTGNSPPVIDNVLDQGQKEE-AAKDCHTRHNSGNGN-----VPLLENROKSF I KVDG GRSPTGNTPPVIDSVSEKGVVNGKDSKE IOEKONPGNGS-VPVRTIGLENRLNSFFOMDS GRSPTGNTPPVIDNVAEKVSSGNKESKDNQGKPNGGNGSSAPARTVGLENRLNSFIQIDS GRSPTGNTPPVIDSVSEKGSSSIKDSKDTHGKOSVGSG--SPVOTVGLETRLNSFVOVEA GRSPTGNTPPVIDTVSEKGNPNPKDSKDNQGKQNVSNGS-APTRTMGLENRLNSFIQVDP GRSPTGNTPPVIDSVSEKGNPN-KDSKDNQAKQNVGNGS-VPMRTVGLENRLNSF IQVDA GRSPTGNTPPVIDSVSEKANPNIKDSKDNQAKQNVGNGS-VPMRTVGLENRLNSFIQVDA

$$
.:^{*}: .:^{*} \quad: \ldots: \text { : }
$$


XP_783363.3

NP_001137312.1 NP_001084351.1 XP_004949340.1 XP 007497871.1 NP_031488.2 NP_001069454.2 XP 014996065.1 AAA 03586.1

XP_783363.3 NP_001137312.1 NP_001084351.1 XP_004949340.1 XP_007497871.1 NP 031488.2 NP_001069454.2 $\mathrm{XP} 014996065.1$ AAĀ 03586.1

XP_783363.3 NP_001137312.1 NP_001084351.1 XP_004949340.1 XP 007497871.1 NP_031488.2 NP 001069454.2 XP-014996065.1 $\mathrm{AA} \overline{\mathrm{A}} 03586.1$

XP 783363.3

NP_001137312.1 NP 001084351.1 XP 004949340.1 XP_007497871.1 NP 031488.2

NP_001069454.2 XP_014996065.1 AAĀ 03586.1

XP 783363.3

NP_001137312.1 NP 001084351.1 XP_004949340.1 XP_007497871.1 NP 031488.2 NP_001069454.2 XP 014996065.1 $\mathrm{AA} \overline{\mathrm{A}} 03586.1$

XP 783363.3

NP_001137312.1 NP 001084351.1 XP_004949340.1 XP 007497871.1 NP_031488.2 NP_001069454.2 XP 014996065.1 AAĀ03586. 1

XP_783363.3 NP 001137312 .1 NP 001084351.1 XP_004949340.1 XP 007497871.1 NP_031488.2 NP 001069454.2 XP 014996065.1 $\mathrm{AA} \overline{\mathrm{A}} 03586.1$
YDKKETERNSGESDSVS------------KSDLSSD-----DOKSSOLKAK--------LDKKVTDIKPAPS-NPNIGPELHEFPVSERTPFSSTNSSKHSSPSGAVAARVSPFNYTPS LDTKGTDPKSLINN----QQETNENTVAERTAFSSSSSSKHSSPSGTVAARVTPFNYNPS PDKKGNETKPLQT-NPVPAPENNESTVSERTPFSSSSSSKHNSPIGAVAARVTPFNYNPS PDKKAAETKSGOV-NLVPAPETSETSVAERTPFSSTSSSKHSSPSGTVAARVTPFNYNPS PEQKGTEAKPGQS-NPVSIAETAETCIAERTPFSSSSSSKHSSPSGTVAARVTPFNYNPS PDQKGTETKPGHSNNPVPASETSESSIAERTPFSSSSSSKHSSPSGTVAARVSPFNYNPS PDOKGTETKPGO-NNPVPVSETNESSIVERTPFSSSSSSKHSSPSGTVAARVTPFNYNPS PDQKGTEIKPGQ-NNPVPVSETNESSIVERTPFSSSSSSKHSSPSGTVA

: * : :

$:: \quad: *$

.. $\quad$ : *

\section{* :}

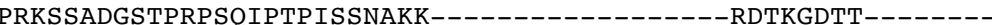
PRKSNGENSTSRPSQIPTPVTNSTKK--------------RDSKTETTDSSGSQSP RRKSSVDNSSARPSOIPTPVNNSTKK-----------------RDSKSENTDSSGTOSP PRKSSADSSAARPSQIPTPVNNSTKK----------------RDSKTENTESSGTQSP PRKSSADSTSARPSOIPTPVSTNTKK-----_--_--------RDSKTDSTESSGAOSP PRKSSTDGTSARPSOIPTPVSNNTKK---_-_-_------RDSKPDSTEPSGTOSP PRKSSADSTSARPSQIPTPVNNNTKK---------------RDSKTDSTESSGTQSP

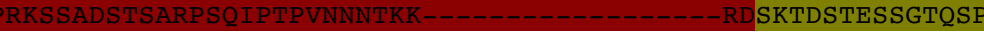

$$
.: \quad *: \ldots
$$$$
\text { : : . }
$$

SRHSSSYSLSSISTNHQVGSLSRQKSAQPELPSSKKSPAVNTRRNIFSSLNKNSNSKSSS

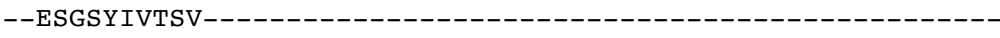

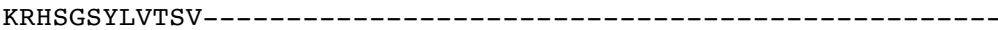

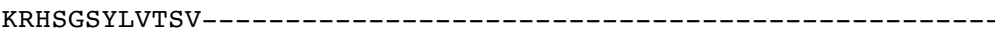

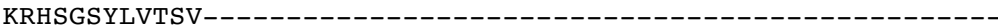

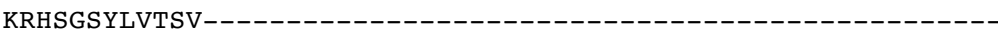

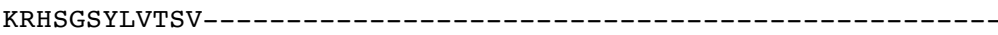

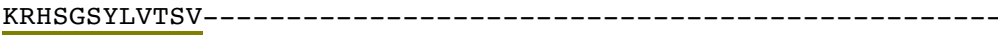

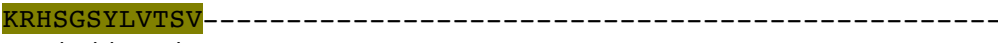
${ }^{*} . * *:: *$ :

2814 2754 2829 2841

2842

2842

2851

2843

2843

KSSLDSSKSGGKSTPKMTSKOKHESEAKPEEKKDKKSEKKRFSFLKLKIGGKKSDSDSEC

KGKSKGFFSKKEKKSPKNLKVQTKSRSESDSMAEHVLPSPPLAELEPDLNMSGADLNFDR 2934 


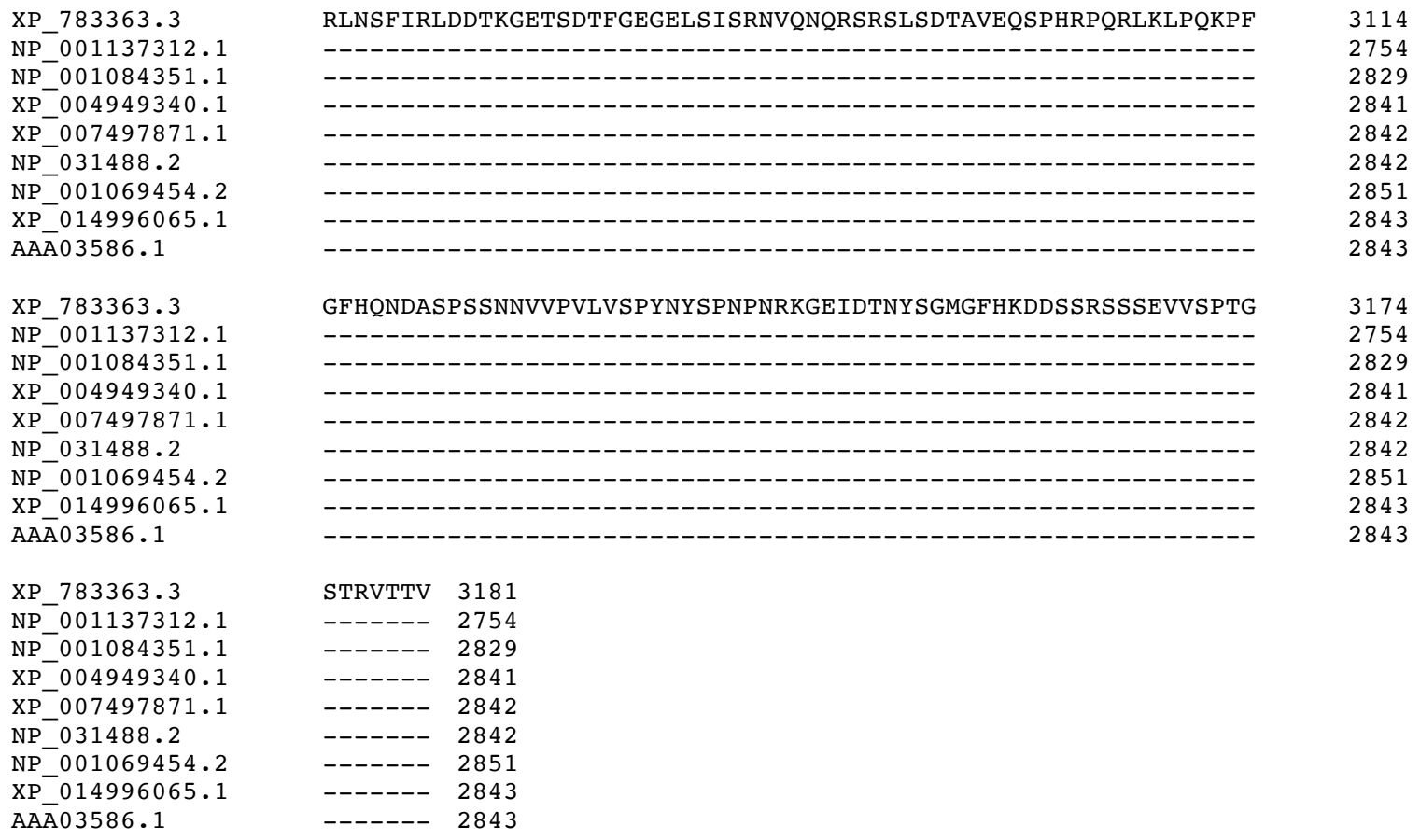

3114

2754

2841

842

2842 843

174

2841

2842

2843

2843

XP_783363.3

NP 001137312 .

NP 031488.2

AAA 03586.1

------ 2843 